\title{
Orquestração de Serviços por Meio de Agentes de Software no Domínio de Vida Ambiente-Assistida
}

\author{
Vinicius Uriel C. Nunes ${ }^{1}$, Vander Alves ${ }^{1}$ \\ ${ }^{1}$ Departamento de Ciência da Computação - Universidade de Brasília (UnB) \\ Campus Universitário Darcy Ribeiro, Instituto Central de Ciências - Brasília \\ DF - Brasil \\ \{viniciusuriel@gmail.com, valves@unb.br\}
}

\begin{abstract}
Solutions in the Ambient Assisted Living domain are inherently complex, resulting that information systems in this domain often require the combination of several modeling strategies. Due to the critical nature of this application domain, the traceability of stakeholders goals within the underlying software is a key modeling issue. To address this problem, this paper presents a preliminary system modeling approach in that domain combining multi-agent systems with service-oriented architecture.
\end{abstract}

Resumo. Soluções em Vida Ambiente-Assistida (VAA) são inerentemente complexas, resultando em que sistemas de informação nesse domínio frequentemente requererem a combinação de diversas estratégias de modelagem. Devido à natureza crítica deste domínio de aplicação, a rastreabilidade dos objetivos dos atores no software subjacente é uma questão-chave para a modelagem. Para resolver este problema, apresentamos uma abordagem preliminar de modelagem de sistema nesse domínio combinando sistemas multi-agentes com arquitetura orientada a serviços.

\section{Introdução}

O processo de envelhecimento da população tem se intensificado na última década nos países industrializados [ONU 2001, Camarano 2002]. As despesas com saúde para os governos e para a sociedade têm aumentado devido à necessidade de tratamento constante para as pessoas idosas ou com necessidades especiais. Com o passar do tempo, a vida social dessas pessoas e as atividades diárias ficam cada vez mais comprometidas, o que diminui a qualidade de vida das mesmas [Nehmer et al. 2006].

Vida Ambiente-Assistida (VAA) [Gross et al. 2009, Nehmer et al. 2006] é uma solução de ambiente inteligente que visa propiciar melhor qualidade de vida para essas pessoas e reduzir os custos com saúde. Essas soluções são compostas por dispositivos e serviços para auxiliar as atividades diárias da pessoa assistida. Soluções em VAA são sistemas de informação críticos e, devido a isso, é especialmente importante assegurar que os objetivos do sistema sejam plenamente alcançados. Em particular, é preciso garantir que os objetivos dos atores sejam representados com precisão nessas soluções. Entretanto, rastrear tais objetivos às funcionalidades do sistema é um problema desafiador uma vez que os requisitos correspondentes aos objetivos dos diversos atores frequentemente estão dispersos em diversos pontos do sistema [Becker et al. 2008]. 
Para resolver este problema, apresentamos um estudo de viabilidade [Shull et al. 2001] de uma abordagem de modelagem de sistema nesse domínio combinando sistemas multi-agentes [Weiss 1999] com arquitetura orientada a serviços [Papazoglou et al. 2007], onde cada ator é representado por um agente que invoca serviços para cumprir seus objetivos facilitando, assim, a rastreabilidade dos objetivos dos atores no sistema. A descrição do domínio de aplicação e caracterização do sistema modelado será apresentada na Seção 2. Em seguida (Seção 3), serão apresentadas a identificação dos serviços, a modelagem dos agentes, e as decisões relevantes de cada uma dessas fases. Posteriormente, na Seção 4, serão discutidos os resultados da modelagem e as lições aprendidas. A Seção 5 aborda trabalhos relacionados. Por fim, as conclusões são apresentadas na Seção 6.

\section{Vida-Ambiente Assistida}

Soluções em VAA são soluções que prestam serviços tais como tratamento de emergências (prevenção, previsão e detecção), melhoria na autonomia das pessoas assistidas e conforto (ex: logística e entretenimento).

A natureza do problema exige que essas soluções apresentem características tais como baixo custo, capacidade de customização, utilização eficiente de recursos (energia elétrica, largura de banda etc.), extensibilidade, usabilidade adequada ao perfil de usuário e confiabilidade [Gross et al. 2009]. Combinar essas caraterísticas é uma tarefa de difícil solução tendo em vista que o favorecimento de uma pode implicar o relaxamento de outra.

O sistema motivador da abordagem de modelagem apresentada neste artigo é o EMERGE (Emergency Monitoring and Prevention) ${ }^{1}$ e tem como principais objetivos a prevenção, previsão e o tratamento de emergências [Gross et al. 2009]. As situações de emergências são divididas em desvios de longo prazo e emergências agudas. Uma emergência aguda é uma situação de emergência que não pode ser prevista, por exemplo um acidente doméstico. Desvios de longo prazo são possíveis situações de emergências detectadas por meio de desvios no padrão de comportamento da pessoa assistida.

Por meio de dispositivos embutidos no ambiente, o sistema coleta dados sobre as atividades diárias da pessoa assistida tais como frequência de uso do banheiro, número de refeições, quantidade de horas de sono, etc. Além disso o sistema é capaz de coletar outros dados tais como sinais vitais, localização da pessoa dentro de sua residência e quedas.

Por meio do histórico dos dados das atividades diárias da pessoa, o sistema consegue detectar desvios no comportamento e notificar o médico da família e seus parentes. Em situações de emergência aguda, o sistema envia notificações para o serviço médico de despacho de emergências. Esse serviço é responsável por confirmar as situações de emergência, encaminhar as emergências para as equipes de atendimento médico de emergência e por acionar serviços de apoio social [Gross et al. 2009]. O EMERGE possui vários usuários desempenhando papéis diferentes. A seguir, serão apresentados alguns desses papéis:

- Pessoa Assistida: esse é o papel desempenhado pela pessoa que está sendo monitorada pelo sistema. O sistema não deve requerer nenhuma habilidade especial

\footnotetext{
${ }^{1}$ www.emerge-project.eu
} 
dessa pessoa e também deve permitir que ela peça ajuda diretamente.

- Centro de despacho: essa central recebe os alarmes de emergência aguda, interpreta-os e aciona as equipes necessárias.

- Serviço sócio-médico: essa central recebe os alarmes de desvios de longo prazo e decide quem deve ser acionado para prestar assistência à pessoa assistida.

- Sistema de detecção de emergência: Unidade de software responsável por monitorar a pessoa assistida e dar o devido encaminhamento às situações de emergência detectadas.

A Figura 1 apresenta uma visão do sistema EMERGE representando os participantes e algumas funcionalidades. A figura apresenta a pessoa assistida em sua casa. Essa casa é equipada por vários sensores que compõem uma rede de sensores. Os dados desses sensores são então agregados em situações mais representativas para o contexto. As situações detectadas pelos perceptores são então repassadas à camada de detecção de emergência que decidirá se as situações informadas representam algum tipo de emergência de acordo com sua base de informações. A camada de interação é responsável por gerir as interações com o usuário e por tomar decisões de acordo com o tipo de evento encaminhado pela camada de detecção de emergências. As emergências e os desvios são encaminhados para o serviço sócio-médico ou centro de despacho, serviços externos à casa da pessoa assistida.

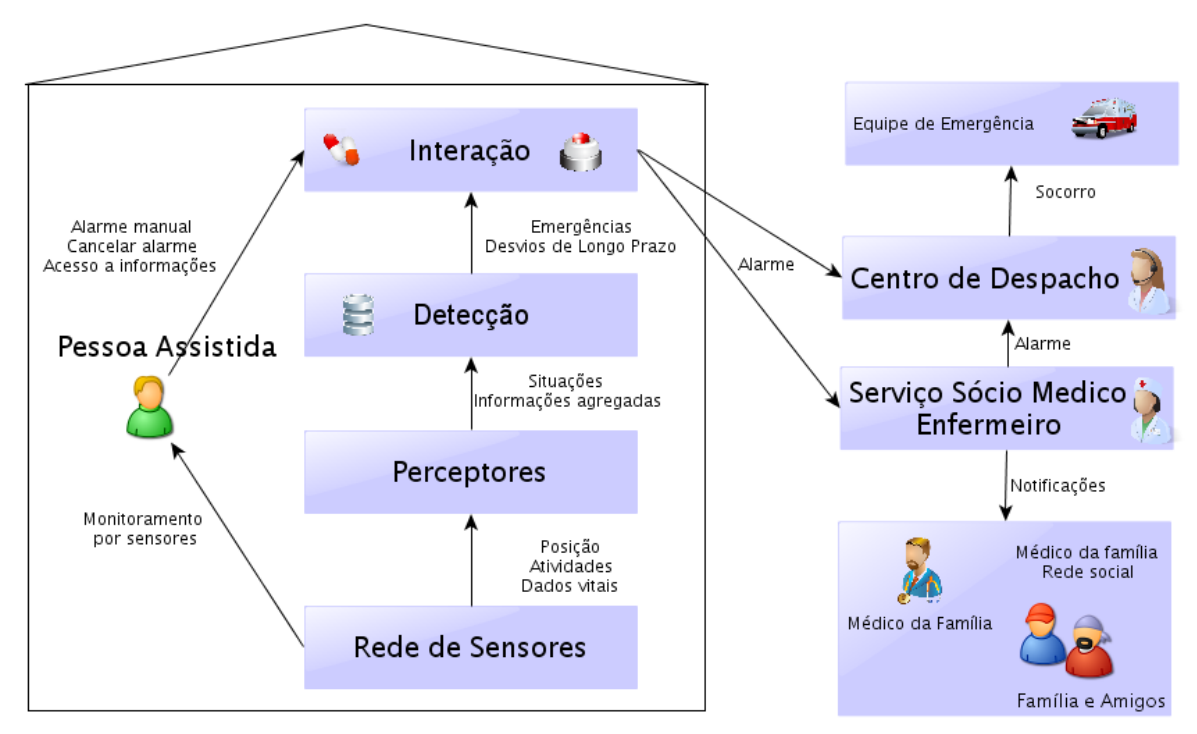

Figura 1. EMERGE - Sistema VAA (adaptado de [Gross et al. 2009])

\section{Modelagem do Sistema por meio de Agentes e Serviços}

A abordagem de modelagem apresentada neste artigo contempla a camada de interação apresentada do EMERGE na Figura 1. Essa camada é responsável por tomar decisões de acordo com os objetivos dos atores, sendo assim a camada mais importante para o problema apresentado. Na modelagem original do EMERGE, esta camada foi modelada segundo análise e projeto orientado a objetos [Becker et al. 2008]. No entanto, conforme relatado pelos autores da proposta, houve uma dificuldade em se rastrear os objetivos dos atores segundo esta abordagem. Desta forma, a proposta deste artigo é definir uma abordagem de modelagem desta camada como um sistema multi-agentes em que cada agente 
representa um ator e os recursos disponíveis aos agentes são os serviços, ou seja, o meio é modelado como um conjunto de serviços que são utilizados pelos agentes para realizar as ações que são desencadeadas a partir de eventos de emergência. Dessa forma, os agentes são os responsáveis pela orquestração dos serviços disponíveis na aplicação. Essa decisão ajuda a administrar a complexidade inerente aos sistemas VAA, já que os atores e o ambiente podem ser modelados de forma mais próxima possível à realidade. Assim, essa abordagem facilita o rastreamento dos objetivos dos atores e a tomada de decisões por meio de analogias com o mundo real. Por exemplo, facilita a resposta de questões tais como: "O sistema deveria fornecer tais dados aos médicos de emergência?” [Becker et al. 2008].

Nesse contexto, os dois atores são a pessoa assistida e o próprio subsistema de deteção de emergência. Esses atores interagem por meio de um fluxo de atividades prédefinido. A Figura 2 apresenta os agentes e os serviços resultantes da modelagem. Os agentes alcançam seus objetivos por meio da invocação dos serviços e pela comunicação com os demais agentes no sistema por meio de protocolos pré-estabelecidos. A seguir serão apresentadas as etapas da modelagem e os resultados obtidos.

\subsection{Cenário exemplo}

Em um cenário típico de emergência, o agente é informado pelo serviço DetectarEmergencia sobre uma possível situação de emergência. O próximo passo do assistente de emergência é tentar confirmar a emergência junto ao assistente pessoal que por sua vez interage com a pessoa assistida por meio do serviço ConfirmarEmergencia para obter a confirmação ou não da situação de emergência. Supondo um cenário de uma emergência real, o usuário irá confirmar a emergência ou a emergência será confirmada automaticamente (timeout) levando o assistente pessoal a enviar a resposta ao agente de emergência. $\mathrm{O}$ assistente de emergência envia, então, os dados da emergência para a central de despacho de ambulâncias (EnviarDadosDespacho) e informa o assistente pessoal sobre o envio de informações a terceiros. As interações entre os assistentes citadas no cenário são as que estão representadas na Figura 2 de cima para baixo, respectivamente.

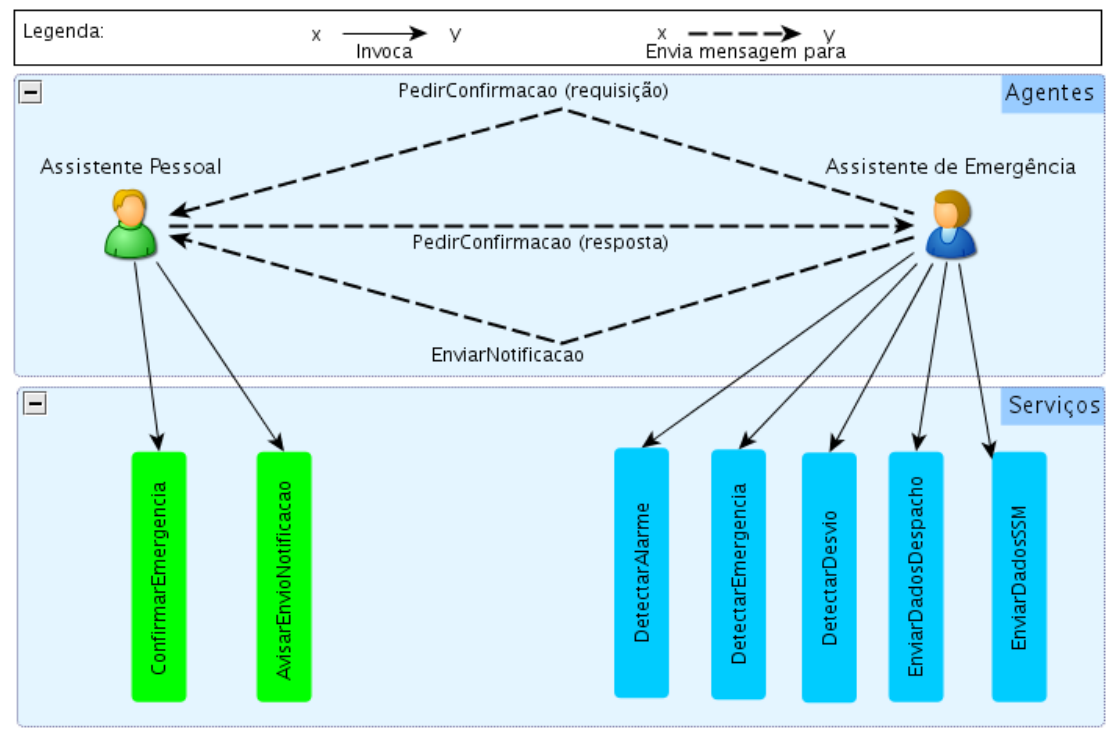

Figura 2. Modelagem dos agentes e serviços 


\subsection{Identificação dos serviços}

A metodologia utilizada é baseada em um método de identificação de serviços a partir da modelagem de processos de negócio [Azevedo et al. 2009]. Antes da execução do método é necessário levantar algumas informações necessárias tais como escopo da identificação dos serviços e modelos de processo utilizados. Assim, a metodologia foi dividida nas seguintes fases de levantamento e identificação.

\subsubsection{Levantamento}

Durante a fase de preparação, os fluxos de processos que representam as atividades desempenhadas pelo sistema ao tratar uma situação de emergência foram detalhados contemplando algumas informações do modelo de atividades tais como papéis, requisitos e regras de negócio. A Figura 3 apresenta o fluxo de atividades obtido representado por meio da notação BPMN [OMG 2009]. A numeração das atividades no fluxo não representam ordenação, são utilizadas apenas para facilitar referências posteriores.

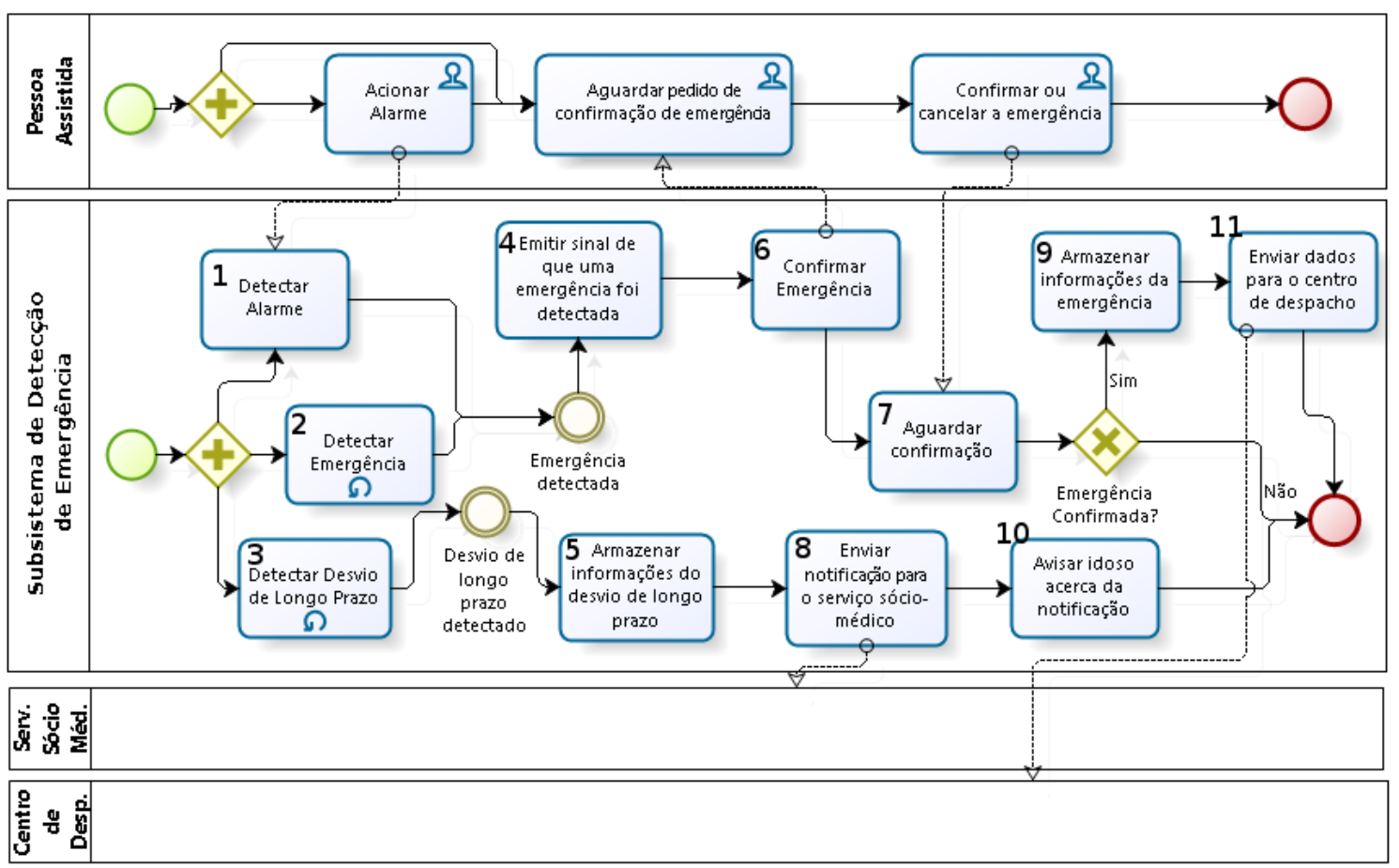

Figura 3. Fluxo de atividades

\subsubsection{Identificação}

Primeiramente, são selecionadas as atividades automáticas e as atividades assistidas pelo sistema. No fluxo de atividades obtido na fase de levantamento, isso significa desprezar as atividades associadas à "Pessoa Assistida" e considerar as atividades ao "Sistema de detecção de emergência”.

As atividades selecionadas do fluxo de processo detalhado serão avaliadas de acordo com as heurísticas apresentadas no trabalho de [Azevedo et al. 2009]. Essas 
heurísticas consideram a semântica do serviço (Heurísticas 1 e 2) e a estrutura do fluxo de processo (padrões no workflow) para identificar os serviços. Um mesmo subconjunto de atividades do processo pode ser identificado por mais de uma heurística. Para evitar ambiguidade e duplicação, as atividades são avaliadas primeiramente pelas Heurísticas 1 e 2 (semântica) e caso não tenha sido identificado nenhum serviço candidato, são utilizadas as heurísticas baseadas em padrões de workflow (as demais). As heurísticas semânticas foram consideradas de maior relevância para a identificação dos serviços uma vez que mapeiam diretamente objetivos dos atores. Por exemplo, o serviço AvisarEnvioNotificacao representa um requisito de negócio: "A pessoa assistida deve ser informanda sempre que forem enviadas informações para outros sistemas" [Gross et al. 2009]. Esse requisito é representado pela atividade número dez do fluxo de atividades.

Em seguida os serviços candidatos foram detalhados na etapa de consolidação, os resultados obtidos dessas etapas estão apresentados na Tabela 1. Esse detalhamento foi feito por meio do fluxo de atividades e de documentação adicional descrevendo os casos de uso do sistema e as atividades. A Tabela 1 não apresenta serviços compostos (serviços que utilizam de outros serviços) porque não foram identificados serviços desta natureza. Além disso, por brevidade, a tabela não possui todas as informações resultantes da modelagem. As atividades listadas na coluna "Atividades" da Tabela 1 são as atividades numeradas na Figura 3 que foram utilizadas na identificação de cada serviço da linha correspondente na tabela.

\begin{tabular}{|c|c|c|l|}
\hline Serviço & Heurística & Atividades & Parametros de Entrada/Saída \\
\hline AvisarEnvioNotificacao & Requisito de negócio (2) & 10 & $\begin{array}{l}\text { Mensagem a ser exibida / ne- } \\
\text { nhum }\end{array}$ \\
\hline DetectarAlarme & Requisito de negócio(2) & 1 & nenhum / nenhum \\
\hline ConfirmarEmergencia & Atividades sequenciais (3) & 4,6 e 7 & $\begin{array}{l}\text { Dados da emergência / sim ou } \\
\text { não }\end{array}$ \\
\hline EnviarDadosSSM & Atividades sequenciais (3) & 5 e 8 & Dados de desvio / nenhum \\
\hline DetectarEmergencia & Atividades em loop (7) & 2 & nenhum / nenhum \\
\hline EnviarDadosDespacho & Atividades sequenciais (3) & 9 e 11 & $\begin{array}{l}\text { Dados da emergência aguda / } \\
\text { nenhum }\end{array}$ \\
\hline DetectarDesvio & Atividades em loop (7) & 3 & nenhum / nenhum \\
\hline
\end{tabular}

Tabela 1. Serviços Consolidados

\subsection{Modelagem dos agentes}

Para a modelagem dos agentes foi escolhida a metodologia GAIA [Zambonelli et al. 2003]: GAIA é uma metodologia de propósito geral, idependente da natureza do problema, baseada na metáfora de organizações em que um sistema de software é concebido como uma instanciação computacional de grupos de interação e indivíduos autônomos que desempenham um ou mais papéis. Sistemas simples podem ser modelados como sendo uma única organização. Para sistemas complexos os princípios de modularidade e encapsulamento sugerem uma divisão da organização em diversas sub-organizações.

Sistemas multi-agentes tipicamente estão inseridos em um meio. O meio pode ser composto por elementos físicos tais como entidades e dados, ou virtuais tais como aplicações, sistemas de bancos de dados e serviços. A modelagem do meio é importante 
para as demais atividades de projeto, entretanto a metodologia GAIA não propõe um método geral para realizar essa atividade, pois essa é uma atividade ligada à natureza do problema. A seguir serão apresentadas as três fases da metodologia GAIA e as decisões tomadas em cada uma delas dentro do escopo definido (Seção 3). Ao final serão apresentadas as Tabelas 2 e $3 \mathrm{com}$ as informações levantadas durante modelagem dos agentes.

\begin{tabular}{|c|c|}
\hline Papel & Assistente Pessoal \\
\hline Descrição & Representa a pessoa assistida \\
\hline Resp. liveness & $\begin{array}{l}\left.{\text { (PedirConfirmacao.InvocarConfirmarEmergência.PedirConfirmacao })^{\omega}}^{(\text {EnviarNotificacao.InvocarAvisarNotificação) }}\right)^{\omega}\end{array}$ \\
\hline Resp. safety & TempoConfirmarEmergencia $<20 \mathrm{~s}$ \\
\hline Permissões & ConfirmarEmergência (consumo), AvisarNotificação (consumo) \\
\hline $\begin{array}{l}\text { Protocolos e } \\
\text { Atividades }\end{array}$ & $\begin{array}{l}\text { PedirConfirmacao, ConfirmarEmergência, } \\
\text { InvocarConfirmarEmergência, EnviarNotificacao }\end{array}$ \\
\hline \multirow{2}{*}{$\begin{array}{l}\text { Descrição das } \\
\text { Atividades }\end{array}$} & Invoca o serviço AvisarNotificação disponível no ambiente \\
\hline & $\overline{\text { InvocarConfirmarEmergência }}$ Invoca o serviço confirmar emergência. \\
\hline
\end{tabular}

Tabela 2. Papel: Assistente Pessoal

\begin{tabular}{|c|c|c|}
\hline Papel & \multicolumn{2}{|l|}{ Assistente de Emergência } \\
\hline Descrição & \multicolumn{2}{|c|}{ Representa o sistema, monitorando eventos de emergência } \\
\hline Resp. liveness & \multicolumn{2}{|c|}{$\begin{array}{l}\text { (InvocarDetectarAlarme } \mid \text { InvocarDetectarEmergencia })^{\omega} . \text { PedirConfirmacao. } \\
\text { [InvocarEnviarDadosDispatcher.EnviarNotificacao] }\end{array}$} \\
\hline Resp. safety & \multicolumn{2}{|c|}{$\begin{array}{l}\text { TempoConfirmarEmergencia }<30 \text { s (Larga Margem de delay em relação ao Assistente } \\
\text { Pessoal) }\end{array}$} \\
\hline Permissões & \multicolumn{2}{|c|}{$\begin{array}{l}\text { DetectarAlarme (consumo), DetectarEmergencia (consumo), DetectarDesvio (consumo), } \\
\text { EnviarDadosDispatcher (consumo), EnviarDadosSSM (consumo) }\end{array}$} \\
\hline $\begin{array}{l}\text { Protocolos e } \\
\text { Atividades }\end{array}$ & \multicolumn{2}{|c|}{$\begin{array}{l}\text { PedirConfirmacao, EnviarNotificacao, InvocarEnviarDadosSSM, InvocarDetectarAlarme, } \\
\text { InvocarDetectarEmergencia, InvocarEnviarDadosDispatcher, InvocarDetectarDesvios }\end{array}$} \\
\hline \multirow{5}{*}{$\begin{array}{l}\text { Descrição das } \\
\text { Atividades }\end{array}$} & InvocarEnviarDadosSSM & Invoca o serviço EnviarDadosSSM disponível no ambiente \\
\hline & InvocarDetectarAlarme & Invoca o serviço DetectarAlarme \\
\hline & $\overline{\text { InvocarDetectarEmergencia }}$ & Invoca o serviço DetectarEmergencia \\
\hline & InvocarEnviarDadosDispatcher & Invoca o Serviço EnviarDadosDispatcher \\
\hline & InvocarDetectarDesvios & Invoca o Serviço DetectarDesvios \\
\hline
\end{tabular}

Tabela 3. Papel : Assistente de Emergência

\subsubsection{Análise}

Nessa fase é decidida a divisão do problema em suborganizações, é determinado o modelo do meio e os modelos de papéis e de interações preliminares. A seguir serão comentadas as decisões em cada uma das atividades dessa fase:

- Definição das organizações: o sistemas foi modelado como uma única organização responsável por identificar situações de emergência e encaminhar prontamente as informações. Nenhuma divisão nessa organização se mostrou necessária; o escopo escolhido para o estudo do problema possui objetivos bem definidos e não apresenta as características sugeridas pela metodologia GAIA como indicadores para a subdivisão da organização. 
- Modelo do meio: o meio foi modelado como um conjunto de serviços. Os agentes cumprem seus objetivos através do consumo (ou invocação) de cada um desses serviços. Os serviços que compõem o meio estão listados na Tabela 1 e na Figura 2. Dessa forma caso mais de um agente precise realizar uma mesma operação de negócio bastaria invocar o mesmo serviço. Essa abordagem simplifica, portanto, a modelagem dos agentes e melhora a consistência do sistema já que, dessa maneira, a alteração em um regra de negócio em um serviço é automaticamente refletida em todos os pontos de chamada. Essa característica é especialmente relevante em um sistema de natureza crítica como os sistemas em VAA.

- Modelo de papéis preliminar: foi identificado um papel preliminar para cada ator envolvido no escopo desse trabalho, mais especificamente a pessoa assistida e o subsistema de detecção de emergência. As informações levantadas nesta fase compreendem que recursos do ambiente (serviços) cada papel pode utilizar (permissões), suas responsabilidades e suas atividades. As responsabilidades podem ser dividias em propriedades safety, invariantes que o agente deve respeitar, e liveness, sequência de atividades e protocolos que o agente deve executar na ocorrência de algum evento específico. Chamamos de atividade as ações que um agente pode realizar sem interagir com os demais agentes; chamamos de protocolo uma determinada sequência de troca de mensagens entre agentes. Retomando o cenário apresentado na Seção 3, temos um exemplo de protocolo quando o assistente de emergência pede que o assistente pessoal confirme junto à pessoa assistida uma possível situação de emergência. Ainda nesse cenário, temos a representação das atividades, que nesse sistema consistem em invocações de serviços como por exemplo, ConfirmarEmergencia.

- Modelo de interações preliminar: as interações identificadas e representadas de maneira preliminar foram identificadas com base no fluxo de atividades e nos casos de uso do sistema. Para cada interação são descritas entradas e saídas, descrição e participantes. Algumas dessas informações são complementadas na etapa de projeto detalhado. A seguir estão descritas as duas interações identificadas:

- Pedir confirmação: interação entre o assistente pessoal e o assistente de emergência quando o agente de emergência detecta um evento de emergência que precisa ser confirmado.

- Enviar notificação: caso alguma informação seja enviada ao Serviço de Despacho ou ao Serviço Sócio-Médico pelo assistente de emergência, este deve informar ao assistente pessoal sobre o envio de informações

- Regras organizacionais: Na modelagem desse sistema a única regra organizacional observada foi: o assistente pessoal (Tabela 2) deve ser avisado sempre que um agente enviar informações para um sistema externo.

\subsubsection{Projeto Arquitetural}

Nesta fase a estrutura organizacional é definida de maneira mais detalhada por meio da definição da topologia e do regime de controle dos papéis na organização. Em posse da estrutura organizacional, o modelo de papéis preliminares é então validado e complementado. A seguir estão comentados os resultados de cada uma das etapas dessa fase: 
- Estrutura Organizacional: a estrutura organizacional é definida por sua topologia e pelo regime de controle. A metodologia GAIA sugere que a topologia seja sempre a mais simples possível que seja capaz de lidar com a complexidade de coordenação e de computação. Dessa forma foi escolhida uma topologia sem divisão hierárquica e o regime de controle adotado foi o de especialização de workload, ou seja, cada agente é responsável por desempenhar uma tarefa específica.

- Complementação dos modelos de papéis: nessa etapa o modelo de papéis é complementado descrevendo atividades, permissões e responsabilidades. As Tabelas 2 e 3 apresentam as informações levantadas nesta etapa estão representadas por meio da sintaxe definida pela metodologia GAIA. Em particular, as responsabilidades liveness utilizam a linguagem semi-formal definida pela metodologia para a representação do sequenciamento de atividades e protocolos. $\mathrm{Na}$ linha "Protocolos e Atividades", as atividades têm seus nomes sublinhados e os protocolos não. Neste trabalho, não será apresentado o detalhamento dos protocolos. Na linha responsabilidades estão representados os serviços a que o papel tem permissão de consumo; são considerados permitidos para um papel apenas os serviços listados nessa linha. A última linha apresenta um breve descrição das atividades que consistem em invocações de serviços. Os serviços mencionados nessa tabela são os serviços apresentados na Tabela 1. As Tabelas 2 e 3 possuem detalhes suficientes para descrever o comportamento dos agentes; as responsabilidades liveness de cada um dos agentes descreve o sequenciamento das atividades e dos protocolos de cada um deles. Por exemplo, retomando o cenário da Seção 3, observamos que a expressão de responsabilidade liveness da Tabela 3 descreve a sequência de atividades do agente de emergência nesse cenário: inicialmente o agente aguarda a detecção de uma emergência ou um sinal de alarme (InvocarDetectarEmergencia | InvocarDetectarAlarme), em seguida se comunica com o agente pessoal por meio do protcolo PedirConfirmacao em seguida o agente de emergência salva e envia dos dados ou encerra a sequencia dependendo da resposta recebida por meio do protcolo PedirConfirmacao.

- Complementação dos modelos de interações: nessa etapa o modelo de interações é então complementado definindo os papéis que participam de cada protocolo.

\subsubsection{Projeto Detalhado}

Nessa etapa, são definidos o modelo de agentes, que associa cada agente a um ou mais papéis definidos na organização, e o modelo de serviços, que identifica os serviços associados a cada agente. Para o modelo de agentes, foi decidido que cada papel seja mapeado a um agente diferente. Essa abordagem cria uma separação de responsabilidades mais evidente, facilitando a compreensão do sistema e a flexibilidade de adicionar ou remover papéis.

O modelo de serviços ${ }^{2}$ apresenta as funções de cada agente; nesse sistema cada agente desempenha um único papel. Sendo assim, os serviços dos agentes são apenas aqueles relacionados às responsabilidades liveness do papel desempenhado. Cada porção dessa propriedade que ocorre em paralelo (separadas pelo símbolo \|| ) é considerada um 
serviço prestado pelo agente. Essas propriedades estão representadas por uma linguagem semi-formal definida pela metodologia GAIA [Zambonelli et al. 2003].

\subsection{Rastreamento Papéis-Serviços}

A Figura 2 apresenta os papéis identificados, as interações entre eles, e os serviços associados a cada papel. A relação entre os serviços e os papéis é determinada da seguinte forma: 1) cada papel representa um ator que por sua vez possui um conjunto de serviços associados; 2) o meio é composto por serviços; 3) as permissões de acesso aos recursos do meio determinam os serviços utilizados por cada papel; 4) as atividades dos papéis representam invocações de serviços; 5) as propriedades liveness determinam a sequência em que os serviços e os protocolos (interações) irão ocorrer.

Por exemplo, retomando o cenário apresentado na Seção 3, temos que o assistente pessoal recebe uma requisição do assistente de emergência para confirmar uma situação de emergência (início do protocolo PedirConfirmacao), em seguida invoca o serviço ConfirmarEmergencia (atividade); esta invocação pode ser realizada pois este serviço faz parte dos conjunto de serviços que podem ser acessados por este papel (permissões). Por fim, o assistente pessoal envia de volta a resposta ao assistente de emergência (fim do protocolo PedirConfirmacao). Repare que o exemplo apresentado corresponde à primeira metade da expressão liveness do papel assistente pessoal (Tabela 2). O restante do cenário pode ser verificado também por meio das informações apresentadas na tabela do assistente de emergência (Tabela 3).

\section{Discussão e Liçoes aprendidas}

A seguir, serão apresentadas e analisadas as lições aprendidas e as decisões importantes tomadas durante a modelagem.

1. Cada ator é representado por um papel na modelagem.

2. Cada papel foi mapeado diretamente para um agente, desta forma, cada agente representa um ator.

3. O meio do sistema multi-agentes foi modelado como um conjunto de serviços.

O Item 1 é interessante do ponto de vista da abstração para modelagem dos agentes facilitando a identificação dos papéis que compõem a organização na metodologia gaia e a delimitação das responsabilidades de cada papel.

O Item 2 é especialmente importante pois dessa forma é possível rastrear facilmente os objetivos dos atores para o sistema, uma vez que o agente associado a cada ator representa os objetivos do ator na camada de decisão da aplicação (Seção 3). Essa característica é especialmente importante para um sistema de natureza crítica pois ajuda a dminiuir as inconsistências entre os objetivos dos atores e o funcionamento do sistema.

O último ponto se mostrou interessante para a integração entre a arquitetura orientada a serviços e o sistema multi-agentes. As responsabilidades dos agentes foram modeladas como uma sequência de invocação de serviços e de participação em protocolos. Dessa forma os agentes são os responsáveis pela orquestração dos serviços. Vale ressaltar

\footnotetext{
${ }^{2}$ Vale ressaltar que os serviços a que a metodologia se refere não são os serviços identificados a partir do fluxo de atividades, que representam os serviços consumidos pelo agente. Os serviços a que a metodologia se refere são os serviços prestados pelos agentes.
} 
que a modelagem dos serviços foi feita independentemente e anteriormente à modelagem dos agentes. Essa abordagem pode ser um gargalo no processo de modelagem com um escopo mais amplo uma vez que a identificação prévia de todos os serviços pode não ser viável.

O modelo de processo utilizado para identificar os serviços descrito na Seção 3.2.2 e apoiado em [Azevedo et al. 2009] está separado por atividades de cada ator o que facilita a visualização das responsabilidades de cada ator. Em modelos mais complexos pode haver compartilhamento de atividades dificultando esse tipo de representação. Em particular, o reuso de serviços na modelagem apresentada traria benefícios do ponto de vista da consistência do comportamento do sistema, uma vez que regras de negócio contidas nesses serviços seriam compartilhadas por todos que os utilizassem evitando a replicação de código no sistema. Além disso, o mesmo método utiliza-se de heurísticas pré-definidas aplicadas sobre o fluxo de atividades. O método proposto por [Azevedo et al. 2009] facilita a rápida identificação dos serviços mas não hierarquiza ou prioriza a utilização de uma heurística em detrimento de outra, deixando a resolução de conflitos a cargo de quem realiza a atividade de identificação. Em especial o uso excessivo de heurísticas que se baseiam em padrões de workflow podem acarretar em serviços pouco representativos do ponto de vista do negócio. Por exemplo, uma mesma atividade pode compor diferentes serviços identificados por heurísticas distintas, o método apresenta de forma clara como resolver esse tipo de conflito.

\section{Trabalhos relacionados}

Os trabalhos relacionados encontrados abordam a combinação de sistemas multi-agentes com arquitetura orientada a serviços para solução de sistemas inteligentes distribuídos. [Lukasz Chomatek 2009] propõe a implementação da arquitetura de agentes especificada pela FIPA por meio de web-services. Nosso trabalho se propõe trabalhar com os dois paradigmas conjuntamente, enquanto o trabalho citado apresenta uma solução para implementar um sistemas multi-agentes por meio de uma arquitetura orientada a serviços. [Luís Ribeiro 2008] propõe a junção de funcionalidades comuns nos dois paradigmas apresentando uma proposta para a realização dessa integração onde aponta fraquezas e benefícios. O foco desse trabalho é automação de sistemas inteligentes distribuídos.

\section{Conclusão}

O rastreamento dos objetivos dos atores para as funcionalidades de um sistema no domínio de VAA é um problema-chave tendo em vista que são sistemas de natureza crítica e é importante assegurar que tais objetivos sejam devidamente representados no sistema. Neste artigo apresentamos um estudo de viabilidade de uma abordagem de modelagem de sistema VAA combinando sistemas multi-agentes com arquitetura orientada a serviços como proposta de solução para esse problema. A combinação dessas tecnologias facilita o rastreamento dos objetivos dos atores na camada de decisão do sistema. Por outro lado, a modelagem proposta aumenta a complexidade do sistema além de requerer que a identificação dos serviços seja realizada previamente à modelagem dos agentes. Como trabalho futuro, este estudo pode evoluir para um estudo observacional e estudo de caso [Shull et al. 2001] objetivando uma avaliação mais precisa dos resultados observados. Além disso, podem ser estudados aspectos de segurança do sistema relativos a 
controle de acesso a informações e as consequências decorrentes do mapeamento dos atores para a implantação desse tipo de controle.

\section{Agradecimentos}

Agradecemos a Ingrid Nunes e a Elder Cirilo por valiosos comentários, e ao Fraunhofer IESE por permitir acesso à documentação técnica do EMERGE.

\section{Referências}

Azevedo, L., Baiao, F., Santoro, F., Souza, J., Revoredo, K., and Pereira, V. (2009). Identificação de Serviços a partir da Modelagem de Processos de Negócio. Anais/SBSI 2009, V Simpósio Brasileiro de Sistemas de Informação, pages 133-144.

Becker, M., Alves, V., Vajda, L., Latour, L., Kyriazanos, D., Mitilineos, S., and Thomopoulos, S. (2008). Emerge architecture design. Technical Report D6.2, Fraunhofer IESE.

Camarano, A. A. (2002). Envelhecimento da população brasileira: uma contribuição demográfica. Technical report, Instituto de Pesquisas Econômicas Aplicada (IPEA).

Gross, A., Steinbach-Nordmann, S., Jedlitschka, A., Becker, M., Steinke, I., and Bloice, M. (2009). Emerge system requirements specification. Technical Report D6.12, Fraunhofer IESE.

Lukasz Chomatek, A. P.-M. (2009). Modern Approach for Building of Multi-Agent Systems . Lecture Notes in Computer Science.

Luís Ribeiro, José Barata, P. M. (2008). MAS and SOA: Complementary Automation Paradigms . IFIP International Federation for Information Processing.

Nehmer, J., Becker, M., Karshmer, A., and Lamm, R. (2006). Living assistance systems: an ambient intelligence approach. In ICSE '06: Proceedings of the 28th international conference on Software engineering, pages 43-50, New York, NY, USA. ACM.

Nunes, V. (2009). Orquestração de serviços por meio de agentes de software no domínio de vida ambiente-assistida. http://monografias.cic.unb.br/dspace/ handle/123456789/225.

OMG (2009). Business process modelling notation. http: / / www . bpmn . org/.

ONU (2001). Population division department of economic and social affairs united nations world population prospects. http://www.un.org/spanish/esa/ population/wpp2000h.pdf.

Papazoglou, M. P., Traverso, P., Dustdar, S., and Leymann, F. (2007). Service-Oriented Computing: State of the Art and Research Challenges.

Shull, F., Carver, J., and Travassos, G. H. (2001). An Empirical Methodology for Introducing Software Processes. In In Proceedings of the 8 th European Software Engineering Conference, pages 10-14.

Weiss, G. (1999). Multiagent Systems, A Modern Approach to Distributed Artificial Intelligence. MIT, United States.

Zambonelli, F., Jennings, N. R., and Wooldridge, M. (2003). Developing multiagent systems: The Gaia methodology. ACM Trans. Softw. Eng. Methodol., 12(3):317-370. 